\title{
Induced superficial chondrocyte death reduces catabolic cartilage damage in murine posttraumatic osteoarthritis
}

\author{
Minjie Zhang, ${ }^{1,2}$ Sriniwasan B. Mani, ${ }^{1}$ Yao He,${ }^{1,3}$ Amber M. Hall, ${ }^{4}$ Lin Xu, ${ }^{5}$ Yefu Li, ${ }^{5}$ David Zurakowski, ${ }^{4}$ \\ Gregory D. Jay, ${ }^{6}$ and Matthew L. Warman ${ }^{1,2}$ \\ 1Orthopaedic Research Laboratories, Department of Orthopaedic Surgery, Boston Children's Hospital and Department of Genetics, Harvard Medical School, Boston, Massachusetts, USA. \\ ${ }^{2}$ Howard Hughes Medical Institute, Boston Children's Hospital, Boston, Massachusetts, USA. ${ }^{3}$ State Key Laboratory of Oral Diseases, Department of Orthodontics, West China Hospital of Stomatology, \\ Sichuan University, Chengdu, China. ${ }^{4}$ Department of Anesthesia, Boston Children's Hospital, Boston, Massachusetts, USA. ${ }^{5}$ Department of Cell Biology, Harvard School of Dental Medicine, \\ Boston, Massachusetts, USA. ${ }^{6}$ Department of Emergency Medicine and School of Engineering, Brown University, and Rhode Island Hospital, Providence, Rhode Island, USA.
}

\begin{abstract}
Joints that have degenerated as a result of aging or injury contain dead chondrocytes and damaged cartilage. Some studies have suggested that chondrocyte death precedes cartilage damage, but how the loss of chondrocytes affects cartilage integrity is not clear. In this study, we examined whether chondrocyte death undermines cartilage integrity in aging and injury using a rapid 3D confocal cartilage imaging technique coupled with standard histology. We induced autonomous expression of diphtheria toxin to kill articular surface chondrocytes in mice and determined that chondrocyte death did not lead to cartilage damage. Moreover, cartilage damage after surgical destabilization of the medial meniscus of the knee was increased in mice with intact chondrocytes compared with animals whose chondrocytes had been killed, suggesting that chondrocyte death does not drive cartilage damage in response to injury. These data imply that chondrocyte catabolism, not death, contributes to articular cartilage damage following injury. Therefore, therapies targeted at reducing the catabolic phenotype may protect against degenerative joint disease.
\end{abstract}

\section{Introduction}

Articular cartilage plays a crucial role in joint motion and load transmission $(1,2)$. The most common disease of articular cartilage, osteoarthritis, affects millions of humans and is a major cause of disability $(3,4)$. Joint injuries (e.g., anterior cruciate ligament and meniscal tears) are increasing in incidence, particularly among young athletes, and predispose individuals to precocious osteoarthritis (5). Inflammatory diseases, such as rheumatoid arthritis, affect approximately $1 \%$ of the population and are another significant contributor to the premature failure of articular cartilage (6). In the United States, more than 4 million persons have had knee-replacement surgery for different cartilage diseases (7). The clinical and financial burden of cartilage disease has prompted substantial research investment into its causes and treatments, including the study of animal models of these disease processes.

Chondrocyte loss and cartilage damage occur with aging and after joint injury (8-12). Although cell death and cartilage failure are correlated, the relationship between chondrocyte loss and cartilage degeneration is unknown. Some studies indicate chondrocyte loss precedes cartilage damage after injury and that chondrocyte repopulation in damaged areas can promote cartilage repair $(13,14)$. Other studies indicate that injured chondrocytes produce factors, such as matrix metallopeptidase 13 (MMP13), ADAM metallopeptidase with thrombospondin type 1 motif 4

Conflict of interest: The authors have declared that no conflict of interest exists. Submitted: July 10, 2015; Accepted: May 13, 2016.

Reference information: J Clin Invest. 2016;126(8):2893-2902. doi:10.1172/JCI83676.
(ADAMTS4), and ADAMTS5, that cause cartilage degeneration (15-18). In this paper, we report on a murine genetic modification that induces chondrocyte death and surgical destabilization of the medial meniscus (DMM) to damage the cartilage surface in order to determine whether chondrocyte death is a cause or consequence of cartilage damage and whether cells that live near the surface of cartilage have the ability to regenerate.

We also describe a confocal microscopic method for measuring cartilage volume and chondrocyte number, distribution, and proliferation in mouse articular cartilage. Samples are prepared and imaged using the confocal microscope, much more easily than when studied using standard histology. Histologic sectioning across the knee joint, the commonly used method for assessing mouse articular cartilage (19), is labor intensive, time consuming, and highly dependent on the investigator's ability to orient, section, and stain specimens to obtain comparable images across animals. We show that confocal imaging of cartilage complements and can supersede existing histologic methods.

\section{Results}

A $3 D$ confocal imaging method for mouse articular cartilage. We imaged $760 \mu \mathrm{m} \times 760 \mu \mathrm{m} \times 50 \mu \mathrm{m}$ regions of 1-month-old, male C57BL/6J mouse humeral heads, femoral heads, medial femoral condyles, and lateral femoral condyles in the orientations depicted (Supplemental Figure 1; supplemental material available online with this article; doi:10.1172/JCI83676DS1). It took approximately 3 minutes to image each cartilage. To assess variability in measurements associated with orienting the samples, we obtained 10 

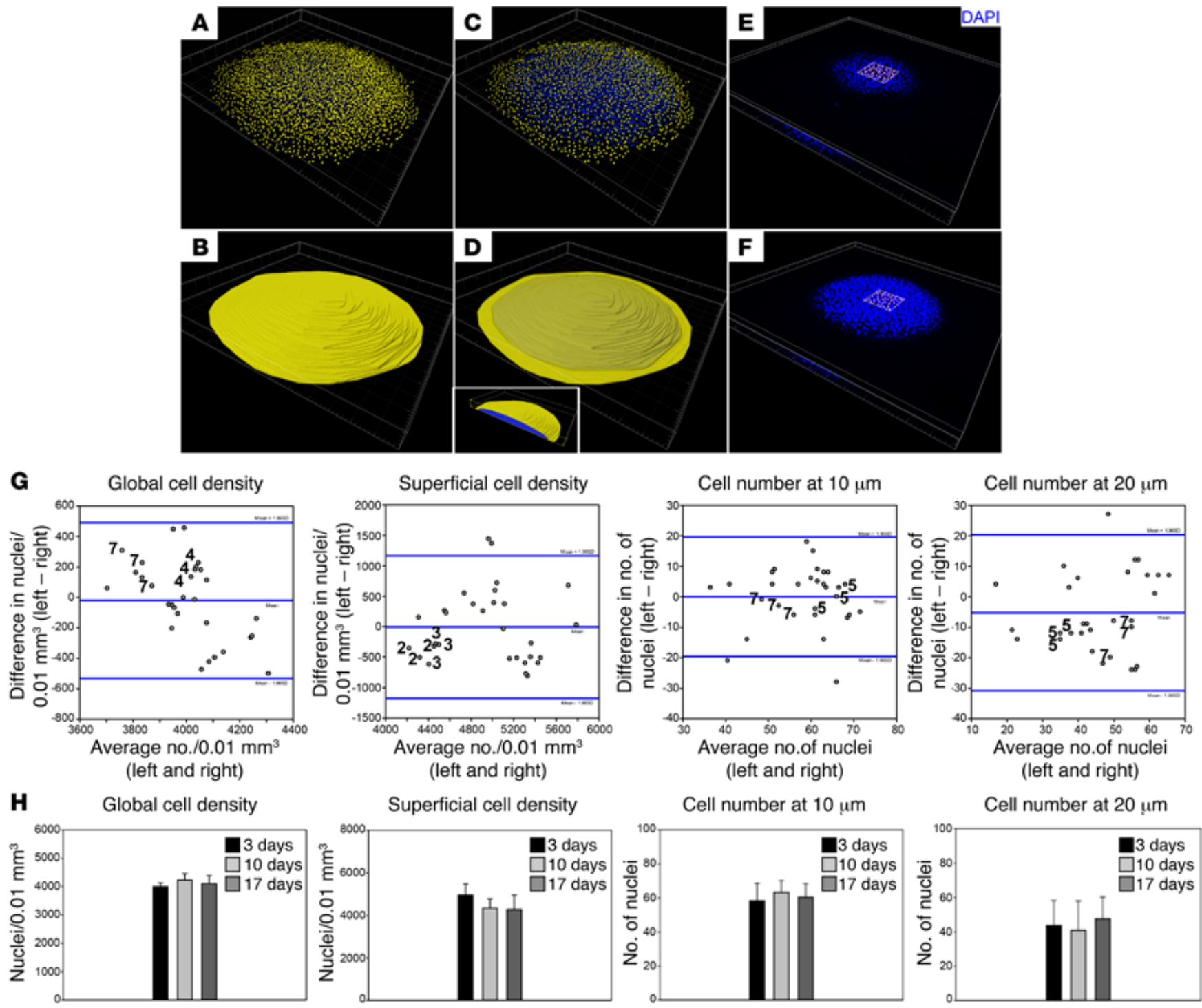

Figure 1. Measuring chondrocyte density and number. (A-F) 3D reconstructed images representing a $760 \mu \mathrm{m} \times 760 \mu \mathrm{m} \times 50 \mu \mathrm{m}$ volume containing a DAPI-stained, wild-type femoral head. The centroids of individual chondrocyte nuclei in the entire cartilage volume (A) and in a volume extending to a depth of $10 \mu \mathrm{m}$ below the surface (C) are indicated by yellow dots. The cartilage volumes across the entire thickness (B) and within the most superficial $10-\mu \mathrm{m}$ region below the surface (D) are colored yellow; the volume covered by the yellow volume in $\mathbf{D}$ is colored blue (inset, $\mathbf{D})$. (E and $\mathbf{F})$ Centroids of chondrocyte nuclei in $100 \mu \mathrm{m} \times 100 \mu \mathrm{m}$ areas (boxes) at depths of $10 \mu \mathrm{m}(\mathbf{E})$ or $20 \mu \mathrm{m}$ (F) are indicated by yellow dots. (G) Bland-Altman plots comparing measures of cell density (number of nuclei/0.01 $\mathrm{mm}^{3}$ cartilage) across the entire cartilage volume (global cell density = chondrocyte nuclei counted in $\mathbf{A}$ divided by cartilage volume as calculated in B), the surface volume extending to a depth of $10 \mu \mathrm{m}$ (superficial cell density = chondrocyte nuclei counted in C divided by surface volume as calculated in D), and cell numbers in the center $100 \mu \mathrm{m} \times 100 \mu \mathrm{m}$ areas at $10 \mu \mathrm{m}(\mathbf{E})$ and $20 \mu \mathrm{m}(\mathbf{F})$ depths for the femoral head. Each circle represents a comparison between contralateral joint surfaces ( $n=10$ mice and 3 comparisons per mouse). Note the circles from an individual mouse cluster near one another. Numbers indicate the median mice with regard to average cell numbers. Blue lines indicate the mean $\pm 1.96 \mathrm{SD}$. (H) Graphs depicting the mean (+ SD) values of the 4 primary measures for the femoral head obtained after storage in fixative for 3 days, 10 days, and 17 days. No significant differences are attributable to the length of time in fixative.

sets of images for each cartilage after having removed and reset the specimen between rounds of imaging (Supplemental Figure 2). We determined that the average of 3 measures in a single specimen provided $95 \%$ CI of falling within 1 SD of the mean from 10 measurements. Therefore, in subsequent experiments, we imaged each cartilage specimen 3 times.

We compared measures of cell number and the number of nuclei in the imaged cartilage volume (cell density) between an individual animal's left and right limbs and between the limbs of different animals. These measurements included the number of chondrocyte nuclei within the entire volume of imaged cartilage (global cell density), the number of nuclei in the cartilage volume making up the first $10 \mu \mathrm{m}$ beneath the surface (superficial cell density), and the number of nuclei in the center $100 \mu \mathrm{m} \times$ $100 \mu \mathrm{m}$ area at $10 \mu \mathrm{m}$ and $20 \mu \mathrm{m}$ beneath the surface (Figure $1, \mathrm{~A}-\mathrm{F})$. Intraanimal variation was less than interanimal variation (Figure 1G, Supplemental Figure 3, and Supplemental Table 1). To determine whether the length of time in fixative affected 

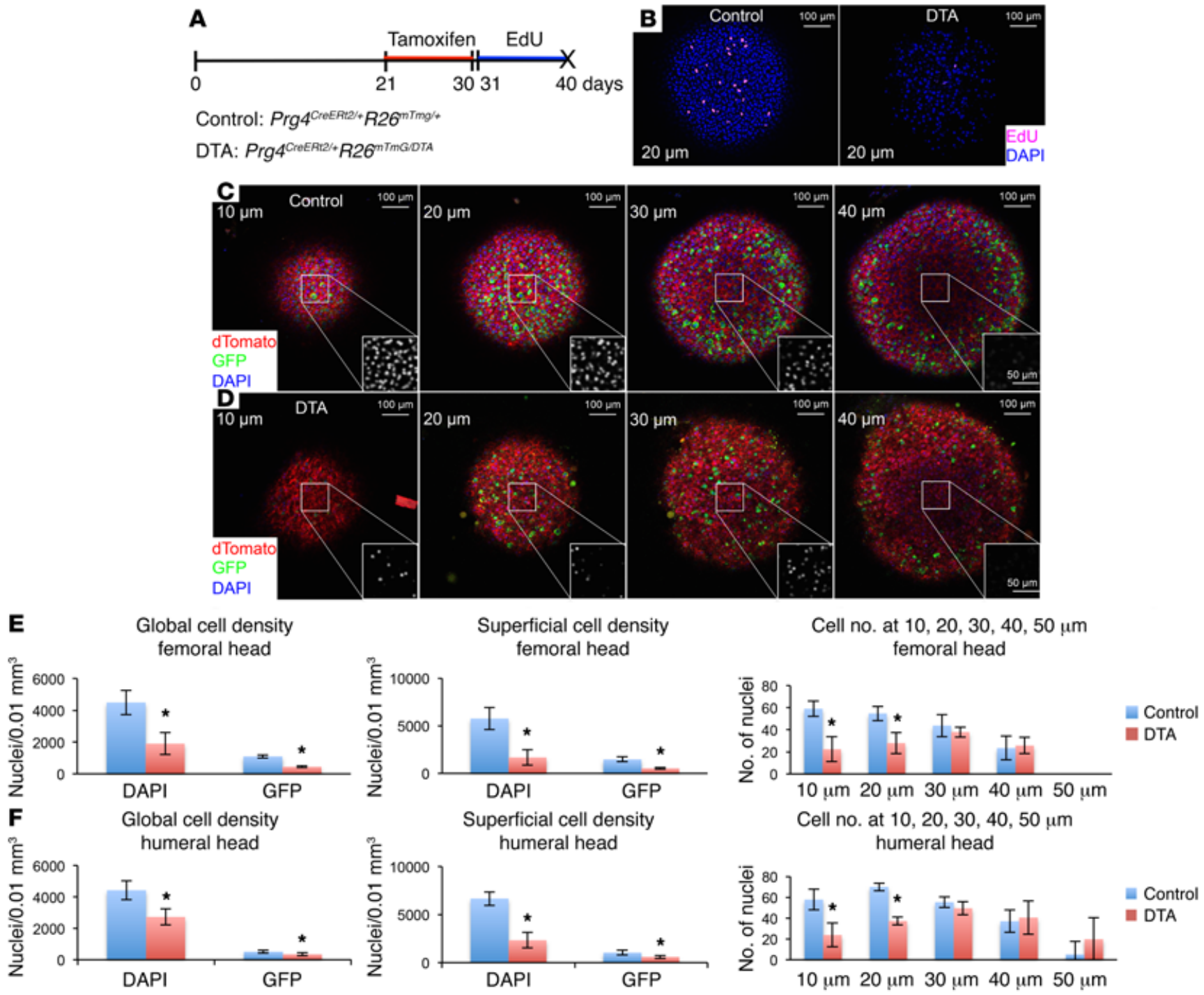

Figure 2. DTA-induced killing of surface chondrocytes. (A) Schematic depicting the experimental timeline for inducing DTA-mediated cell death by administering tamoxifen from 21 to 30 days of age and then monitoring chondrocyte division by administering EdU from 31 to 40 days of age. Animals were analyzed after the final EdU dose. (B) Confocal images showing EdU-positive chondrocyte nuclei in a plane $20 \mu \mathrm{m}$ below the surface of DAPI-stained femoral heads of a control mouse (Prg $4^{\text {CreERt2+ }} R 26^{\mathrm{mTmC/+}}$ mouse) (left) and a DTA-ablated mouse (Prg $4^{\mathrm{CreERtz} /+} R 26^{\mathrm{mTmC} / D T A}$ mouse) (right). Note the decreased numbers of total (blue) and dividing (pink) chondrocyte nuclei in the DTA-ablated mouse. (C and D) Confocal femoral head images taken at different planar depths $(10,20,30$, and $40 \mu \mathrm{m})$ in control mice (C) and DTA-ablated mice (D) identify DAPI-stained chondrocyte nuclei (blue), nonrecombined membraneanchored dTomato-expressing chondrocytes (red), and Cre-recombined membrane-anchored eGFP-expressing chondrocytes (green). Identical thresholds were employed for all images. Insets indicate DAPI windows (pseudocolored white) from a $100 \mu \mathrm{m} \times 100 \mu \mathrm{m}$ area in the center of the image. (E and $\mathbf{F}$ ) Bar graphs depicting the mean $( \pm S D)$ values for chondrocyte density and chondrocyte number for the femoral $(\mathbf{E})$ and humeral $(\mathbf{F})$ heads of control (blue bars) and DTA-ablated (red bars) mice $(n=5) .{ }^{*} P<0.05$, between control and DTA-ablated mice using Student's $t$ test. Scale bar: $100 \mu \mathrm{m}$.

cell number or cell density, we measured the same specimens at weekly intervals (Figure $1 \mathrm{H}$ and Supplemental Figure 4) and observed no systematic differences (Supplemental Figure 4 and Supplemental Table 2).

Based on the aforementioned results, we calculated sample sizes that would be required to detect $10 \%, 20 \%, 30 \%, 40 \%$, and $50 \%$ changes in global and superficial cell density and in cell number within the $100 \mu \mathrm{m} \times 100 \mu \mathrm{m}$ areas (Supplemental Table 3).

Chondrocyte death does not cause cartilage surface damage. Mice that have a cDNA encoding a chimeric GFPCreERt2 protein knocked into the first coding exon of $\operatorname{Prg} 4$ can have Crerecombinase activity induced in most chondrocytes near the surface of articular cartilage (20). We generated mice that have a $\operatorname{Prg} 4^{\text {CreERt2 }}$ allele and a $R 26^{\mathrm{mTmG}}$ allele; the latter allele causes cells to produce membrane-bound tomato fluorescent protein (dTomato) until Cre-mediated recombination causes the cells to produce membrane-bound enhanced GFP (eGFP). We also generated mice with $\operatorname{Prg} 4^{\text {CreERt }}, R 26^{m T M G}$, and $R 26^{\text {DTA }}$ alleles. The last allele causes a cell to produce diphtheria toxin A (DTA) following Cre-mediated recombination, which results in autonomous cell death (21). We administered tamoxifen or vehicle to 3-week-old

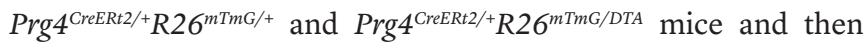
performed confocal microscopy on their articular cartilage 1 week, 2 months, and 8 months later. The Prg $4^{\text {GFPCreERt2/+ }} R 26^{\text {mTMG/DTA }}$ 

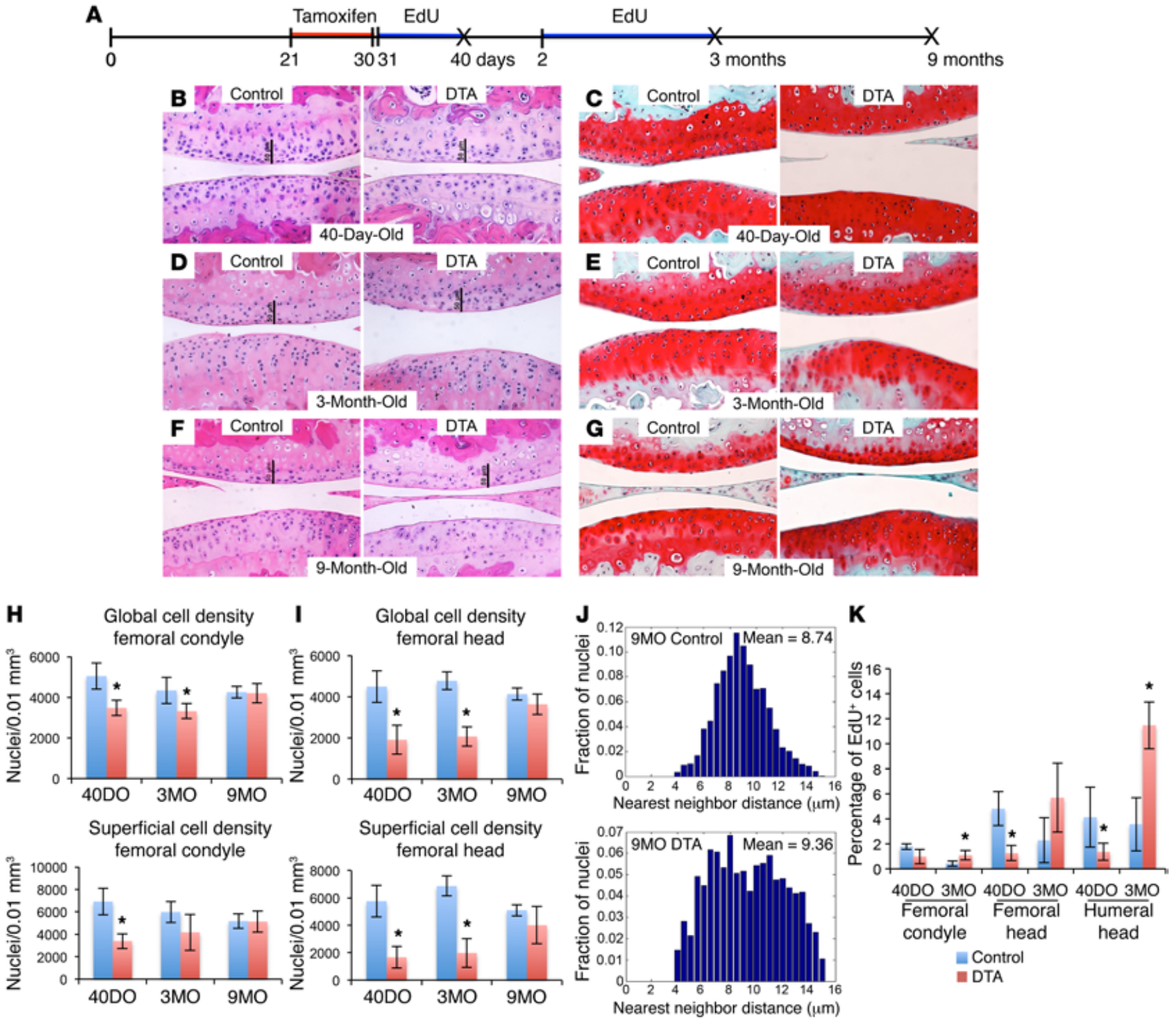

Figure 3. Preservation of cartilage surfaces despite DTA-induced killing of surface chondrocytes. (A) Schematic depicting the experimental timeline for inducing cell death with tamoxifen-mediated induction of DTA expression from 21 to 30 days of age. Subsequent cell division was monitored by administering EdU from 31 to 40 days of age or from 2 to 3 months of age. (B, D, and F) Representative H\&E-stained sagittal sections through the tibial-femoral joint of 40-day-old (B), 3-month-old (D), and 9-month-old (F) control mice (left) and DTA-ablated mice (right) ( $n=5 /$ time point). (C, E, and $\mathbf{G})$ Representative Safranin 0- and Fast Green stained sagittal sections through the tibial-femoral joint of 40-day-old (C), 3-month-old (E), and 9-month-old (C) control mice (left) and DTA-ablated mice (right) ( $n=5 /$ time point). Scale bars: $50 \mu \mathrm{m}$. ( $\mathbf{H}$ and $\mathbf{I})$ Bar graphs depicting the mean ( \pm SD) global cell densities and superficial cell densities in the lateral condyle $\mathbf{( H )}$ and femoral head (I) of control (blue bars) and DTA-ablated (red bars) mice at different time points ( $n=5 /$ time point). Note chondrocyte density measured globally and in the superficial zone decreased significantly in 40-day-old and 3-month-old DTAablated mice compared with controls, with the greatest decrease occurring in the superficial zone. However, this decrease disappeared at 9 months of age. ${ }^{*} P<0.05$, Student's $t$ test. (J) Representative histograms depicting the distribution of nearest neighbor distances for DAPI-stained nuclei in the femoral heads of 9-month-old control (upper) and DTA-ablated (lower) mice. Note the significantly increased mean distance in the DTA-ablated mice $(P<0.001$, Student's $t$ test, $n=5)$ and the significant difference in the histogram shape $(P=0.023$, Levene's test) between control and DTA-ablated mice. (K) Bar graphs depicting mean $( \pm \mathrm{SD}$ ) percentages of DAPI-stained chondrocyte nuclei that are also EdU positive $(n=5)$. Note the DTA-ablated mice (red bars) exhibit significantly lower percentages of EdU-positive cells at 40 days of age compared with control mice (blue bars), but significantly higher percentages of EdU-positive cells at 3 months of age. ${ }^{*} P<0.05$, Student's $t$ test.

mice that received tamoxifen (i.e., DTA ablated) had significantly fewer superficial chondrocytes compared with controls (i.e., $\operatorname{Prg} 4^{C^{\mathrm{CrERR}} 2 /+} R 26^{m T m G / D T A}$ mice that received vehicle only and

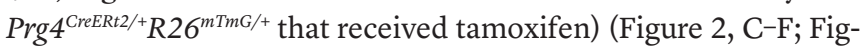
ure 3, B-I; and Supplemental Figure 5). Notably, DTA-induced cell killing did not cause cartilage deterioration, even 8 months later (Figure 3, B-G, and Supplemental Figure 5, A-F). Although $\operatorname{Prg} 4^{4^{\text {CreERt }}}$ is also expressed in synovium and tendon, no abnormal phenotype was observed in these tissues from DTA-ablated mice (Supplemental Figure 6). 

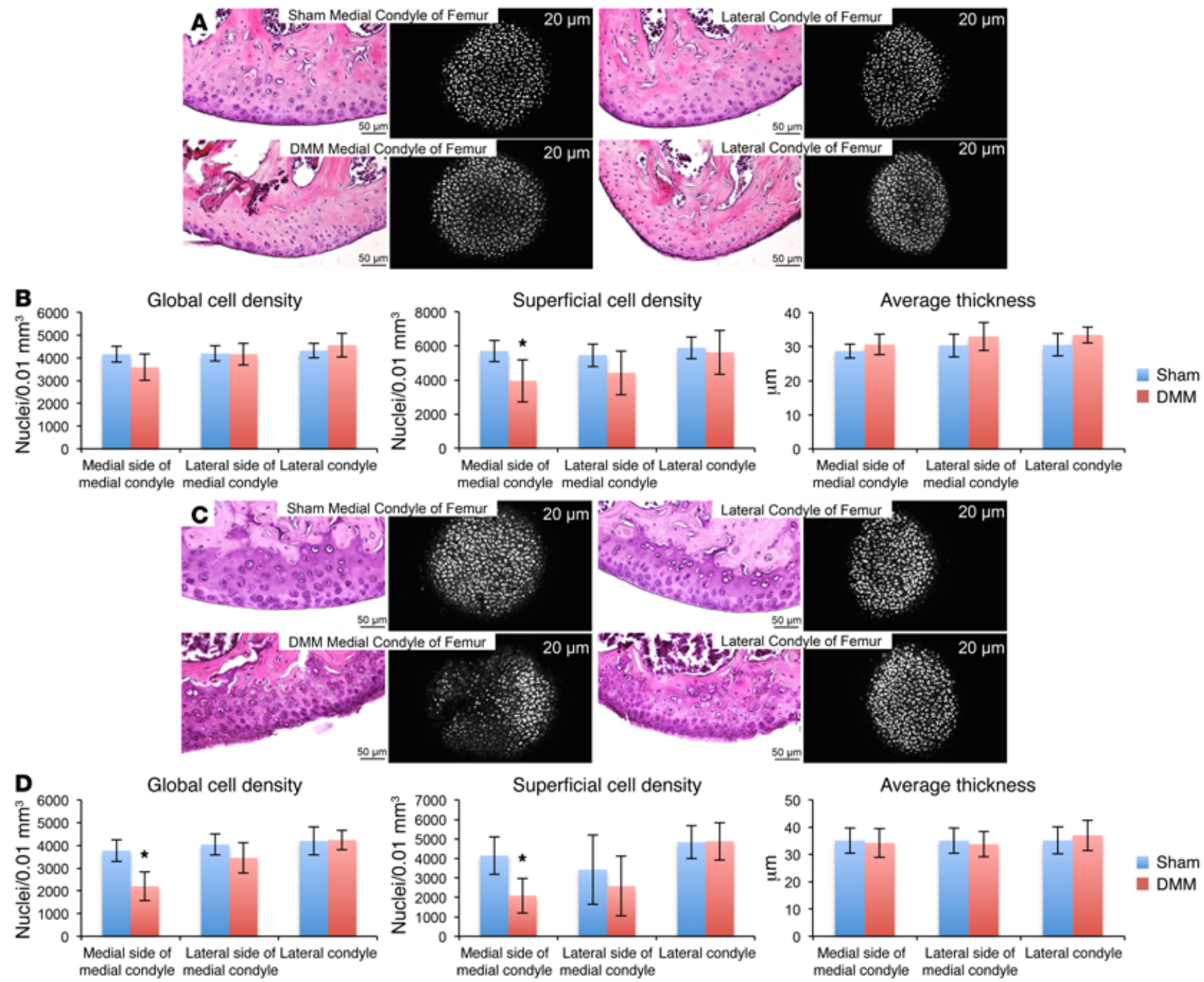

Condyle of Femur
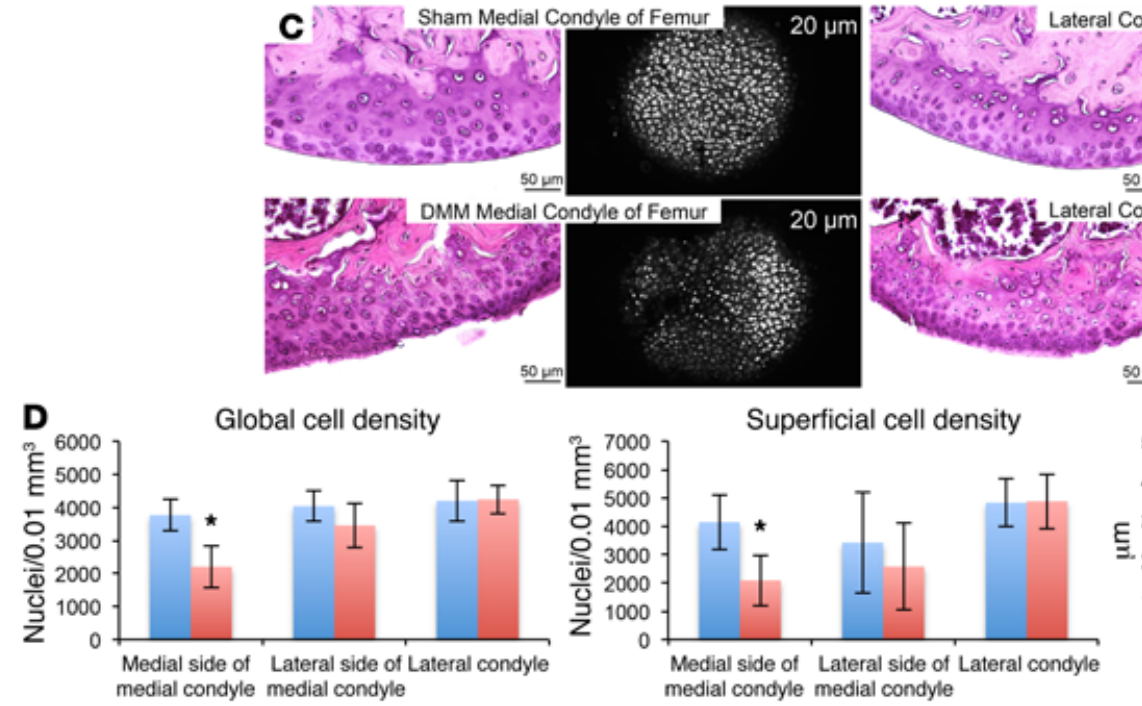
medial condyle medial condyle
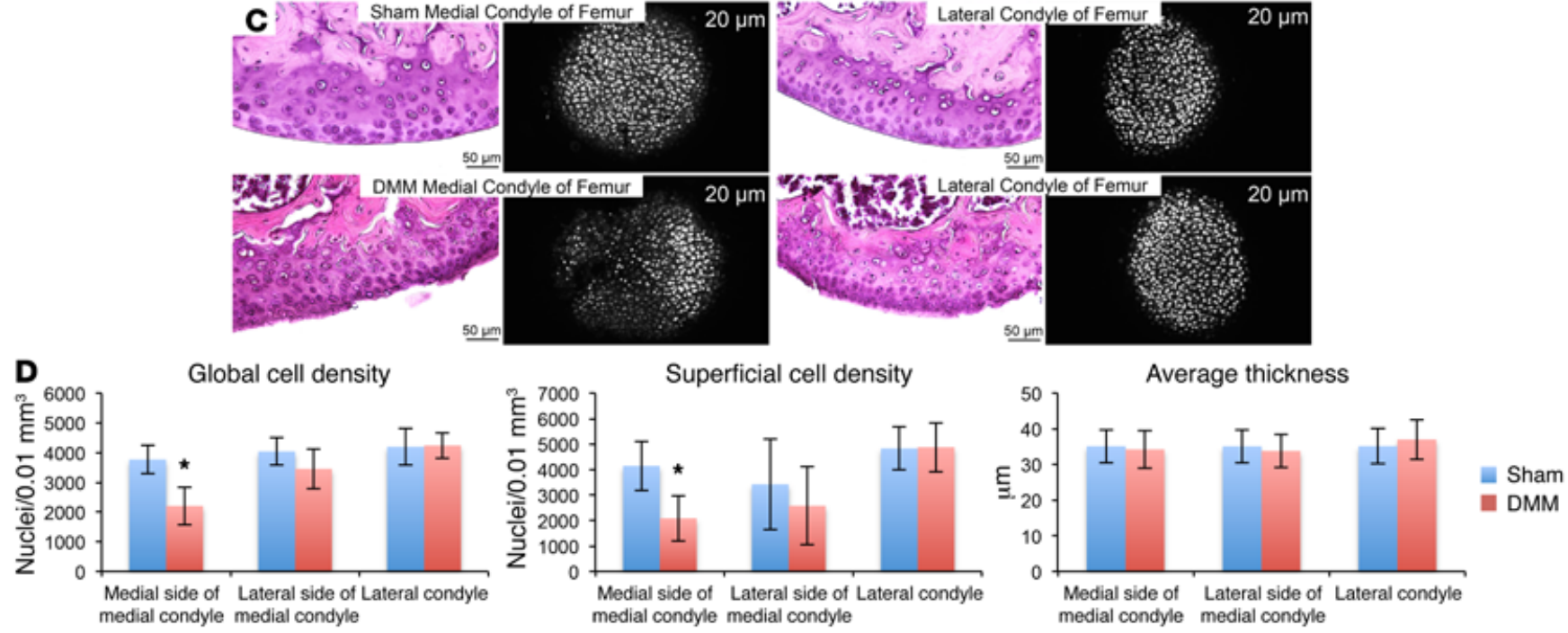

Figure 4. Chondrocyte death following mechanical destabilization in the knee. (A) Representative H\&E-stained coronal sections (left) and confocal images of DAPI-stained nuclei at depths of $20 \mu \mathrm{m}$ (right, pseudocolored white) in medial and lateral condyles of 14-week-old mice that had undergone sham (upper) or DMM (lower) surgery at 10 weeks of age $(n=6)$. (B) Bar graphs depicting the mean $( \pm$ SD) values for global cell density, superficial cell density, and cartilage thickness in condyles from 14-week-old mice that had undergone DMM surgery (red bars) or sham surgery (blue bars) 4 weeks earlier $(n=6)$. Note only the superficial cell density on the medial side of the medial condyle is lower in the DMM compared to shame treated mice. ${ }^{*} P<0.05 \mathrm{Stu}-$ dent's $t$ test. (C) Representative H\&E-stained coronal sections (left) and confocal images of DAPI-stained nuclei at depths of $20 \mu \mathrm{m}$ (right, pseudocolored white) in medial and lateral condyles of 22-week-old mice that had undergone sham (upper) or DMM (lower) surgery at 10 weeks of age ( $n=9$ ). Note the appearance of surface roughening in both condyles and the reduction in the number of DAPI-stained nuclei in the medial, but not in the lateral, condyle of the DMM mice compared with sham-treated mice. (D) Bar graphs depicting the mean ( \pm SD) values for global cell density, superficial cell density, and cartilage thickness in condyles from 22-week-old mice that had undergone DMM surgery (red bars) or sham surgery (blue bars) 12 weeks earlier ( $n=9$ ). Note the significant decrease in chondrocyte density in medial surface of the medial condyle. ${ }^{*} P<0.01$, Student's $t$ test. The medial surface of the medial condyle is normally protected when the meniscus is intact. Scale bar: $50 \mu \mathrm{m}$.

We also used $\operatorname{Prg} 4^{\text {CreRRt2/+}} R 26^{m T m G / D T A}$ mice to induce chondrocyte death at 3 months of age and again observed no deleterious effects on the articular cartilage surface when the mice were 6 months old (Supplemental Figure 7).

Surviving chondrocytes divide following DTA ablation, but their distribution in cartilage remains abnormal. We monitored cell division in articular cartilage by giving 5-ethynyl-2'-deoxyuridine (EdU) to control and DTA-ablated mice (Figure 2A and Figure $3 \mathrm{~A})$. Cell proliferation initially decreased in DTA-ablated mice compared with controls (Figure 2B, Figure 3K, and Supplemental
Figure 8), but within 2 months, the DTA-ablated mice had rates of chondrocyte proliferation that were 2- to 3-fold higher than those of control mice. These data indicate that surviving cells retain the ability to divide (Figure 3K and Supplemental Figure 8). In femoral condyles, the total number of chondrocytes (not shown) and the chondrocyte density in the DTA-ablated 3-weekold mice returned to control values by 9 months of age (Figure $3 \mathrm{H})$. However, the distribution of chondrocytes within the articular cartilage differed significantly between 9-month-old control and DTA-ablated mice (Figure 3J). 

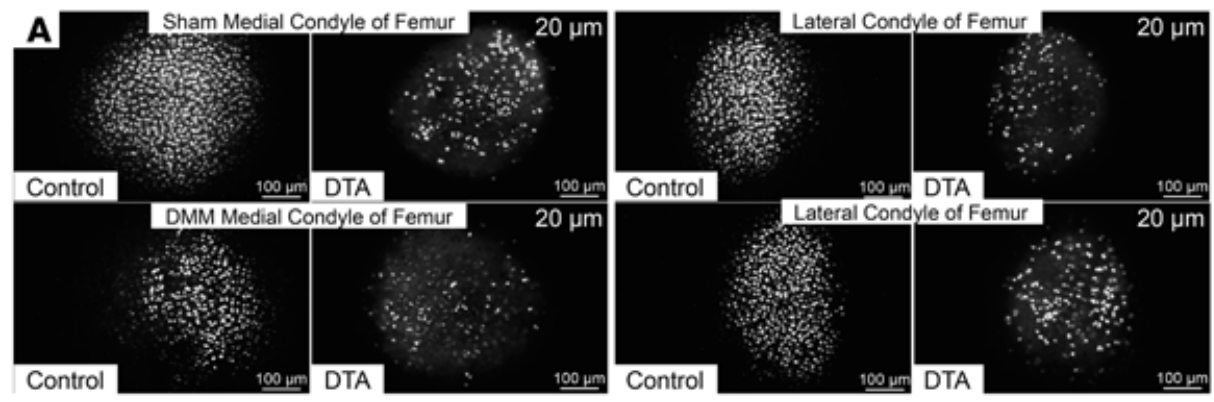

B Medial side of medial condyle global density

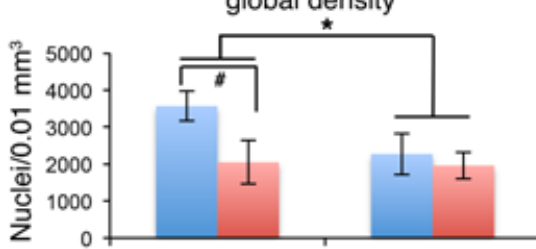

Control

DTA

C Medial side of medial condyle superficial density

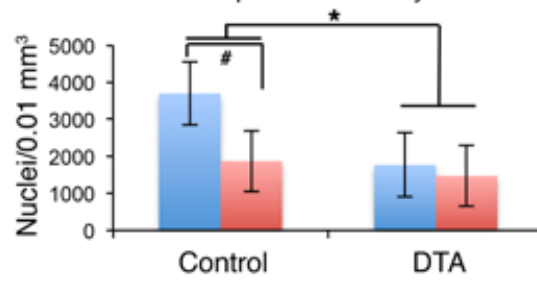

Lateral side of medial condyle global density

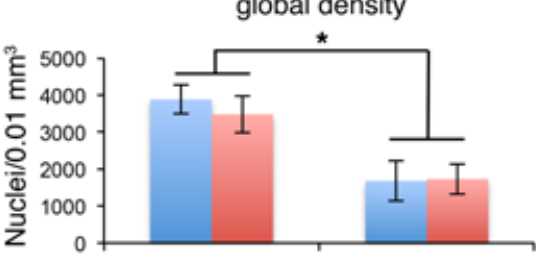

Control

DTA

Lateral side of medial condyle superficial density

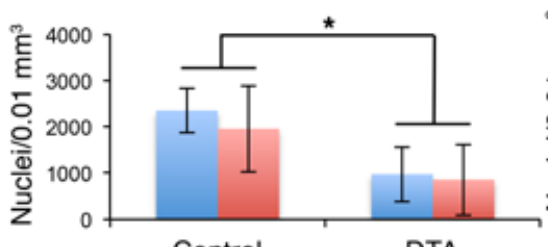

Control

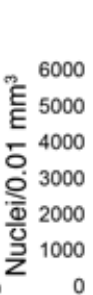

Lateral condyle global density

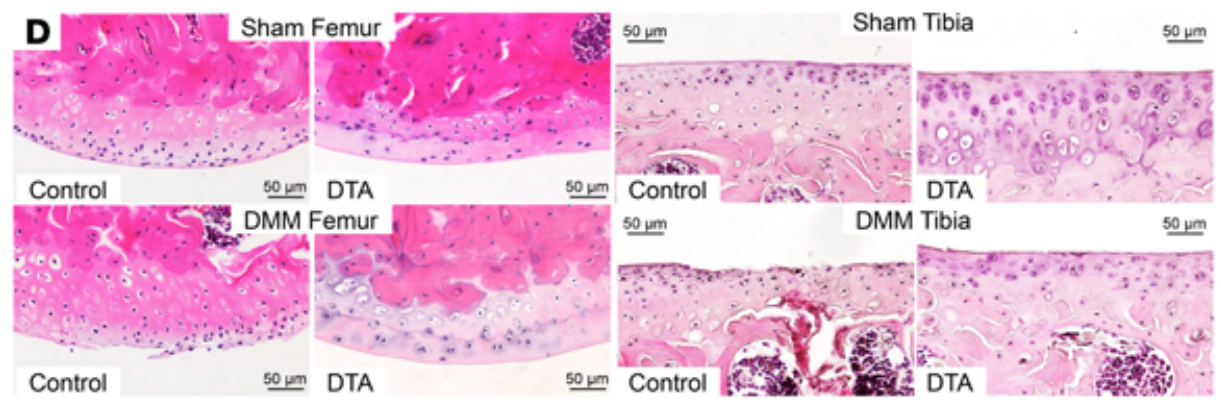

so $\mathrm{\mu m}$

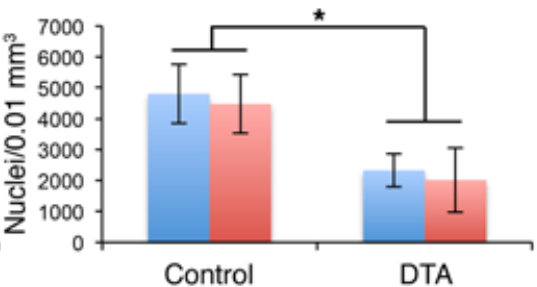

Sham

DMM

Control

DTA

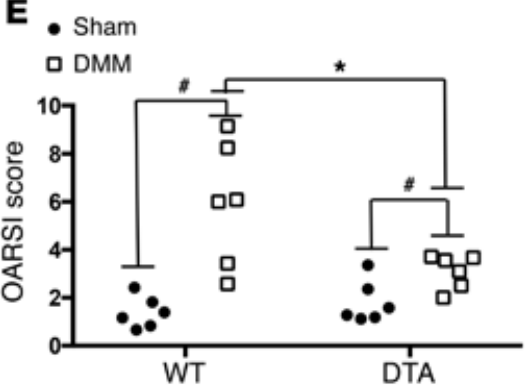

Figure 5. Chondrocyte loss prevents cartilage from being damaged by DMM surgery. (A) Representative confocal images of DAPI-stained nuclei at depths of $20 \mu \mathrm{m}$ (pseudocolored white) in medial and lateral femoral condyles of mice that had undergone sham (upper) or DMM (lower) surgery 12 weeks earlier $(n=6)$. Scale bar: $100 \mu \mathrm{m}$. (B and C) Bar graphs depicting the mean ( \pm SD) values for global cell density (B) and superficial density (C) in condyles from mice that had undergone DMM surgery (red bars) or sham surgery (blue bars) 12 weeks earlier $(n=6)$. Note the significant decrease in chondrocyte density in all condyles of the DTA mice compared with control mice. Also note that DMM surgery did not further affect chondrocyte density in the DTA-ablated mice, whereas cell density decreased significantly in the medial condyles of nonablated mice that underwent DMM versus sham surgery. ${ }^{*} P<0.05$, control vs. DTA, Student's $t$ test. ${ }^{~} P<0.05$, sham vs. DMM, Student's $t$ test. (D) H\&E-stained coronal sections in medial femoral condyles (left) and medial tibial plateau (right) of 22-week-old mice that had undergone sham (upper) or DMM (lower) surgery 12 weeks earlier. Images are from the mouse with the median OARSI score in each group $(n=6)$. Scale bar: $50 \mu \mathrm{m}$. (E) OARSI scores of control or DTA-ablated mice that underwent sham or DMM surgery 12 weeks earlier $(n=6$ /group). Note the significantly higher OARSI score in DMM joints in both control and DTA mice versus sham joints and significantly lower OARSI score in DMM joints in DTA mice versus DMM joints in controls. ${ }^{*} P<0.05$, control vs. DTA, nonparametric Wilcoxon rank-sum test. ${ }^{\#} P<0.05$, sham vs. DMM, nonparametric Wilcoxon rank-sum test.

Chondrocyte death is a consequence of cartilage damage after joint injury. Surgically induced DMM causes osteoarthritis predominantly affecting the medial compartment of the mouse knee within 3 months of surgery (22). Therefore, we examined the medial and lateral condyles of adult mice that had undergone DMM or sham surgery 4 weeks and 12 weeks after the procedure. Four weeks after surgery, there was no cartilage degradation and no change in global cell density in either condyle of the DMM mice compared with the sham-surgery controls (Figure 4, A and B). However, superficial cell density in the medial side of the medial condyle of DMM mice was lower compared with that of controls (Figure 4, A and B). By 12 weeks after surgery, DMM mice had evidence of cartilage damage and a significant decrease in chondrocyte number and density in the medial condyle compared with the sham controls (Figure 4, C and D), but only mild cartilage surface damage and a nonsignificant 
change in chondrocyte number and density in the lateral condyle (Figure 4, C and D).

Living chondrocytes cause cartilage degeneration after injury. Having found that killing chondrocytes did not cause cartilage damage in an otherwise normal joint and that DMM surgery caused cartilage damage and chondrocyte loss, we next determined whether the presence of living chondrocytes reduces or enhances cartilage damage following injury. We performed sham or DMM surgery on 10-week-old mice that had or had not previously undergone DTA ablation. When we examined the joints 12 weeks later, mice whose surface chondrocytes had been DTA ablated before DMM had significantly less cartilage damage than did control mice that underwent DMM (Figure 5). Also, whereas DMM surgery led to loss of chondrocytes in the control mice, it did not further decrease chondrocyte numbers in the DTA-ablated mice (Figure 5, A-C). These data indicate that living surface chondrocytes enhance cartilage damage after DMM, even though these cells ultimately die as a consequence of DMM.

\section{Discussion}

To determine whether chondrocyte death and cartilage damage have a causal relationship, we examined the consequences of conditionally killing chondrocytes at the articular cartilage surface and/or physically destabilizing the medial compartment of the knee joint. We observed no deleterious effect from killing chondrocytes when knee joints were examined as much as 8 months later (Figure 3 and Supplemental Figure 5). In fact, we observed increased rates of chondrocyte cell division in DTA-ablated mice, as measured by EdU incorporation (Figure 3K and Supplemental Figure 8). Thus, DTA-induced death of articular chondrocytes did not initiate cartilage damage. This finding is consistent with work reported by de Crombrugghe and colleagues, which altered chondrocyte identity in mouse articular chondrocytes by conditionally inactivating Sox9 (23). The Sox9-inactivated cells no longer expressed chondrocyte-specific transcripts, yet a year later, the mice had no evidence of cartilage damage (23). Thus, neither survival of chondrocytes nor maintenance of chondrocyte identity is required to protect mouse cartilage over the short term. Human articular cartilage without living cells is also reported to remain free of osteoarthritic changes for several years in cancer patients who had received irradiated, cadaveric osteochondral grafts during limb-salvage surgery (24).

We observed after DTA ablation that surviving chondrocytes retained the ability to divide in vivo (Figure $3 \mathrm{~K}$ and Supplemental Figure 8). This is compatible with earlier observations that articular chondrocytes can be expanded in culture ex vivo (25) and be induced to divide in vivo in disease states, such as osteoarthritis (26). Despite rates of chondrocyte division becoming significantly higher in DTA-ablated cartilage, the distribution of chondrocytes did not return to normal (Figure 3J). This likely reflects the inability of chondrocytes to migrate through cartilage. Thus, areas devoid of chondrocytes remained so and the progeny from chondrocyte divisions remained near one another in a manner similar to that seen in the "cloning" that is observed in osteoarthritic cartilage.

We performed DMM surgery to produce medial compartment instability and ascertain the effect of this injury on chondrocyte viability (Figure 4). DMM alters joint biomechanics so that histo- logic changes resembling osteoarthritis first develop in the medial compartment of the knee joint and secondarily occur in the lateral compartment (22). Other investigators have shown TUNEL staining, an indicator of apoptosis, increases after DMM (27), which is consistent with the cell loss we observed by confocal imaging and histology (Figure 4). These data indicate that cartilage damage, attributable to altered biomechanics in the DMM model, is a proximate cause of chondrocyte loss. Other studies have suggested that material damage to the cartilage surface is another antecedent of chondrocyte death (28-30). For example, depletion of surface lubrication in human cartilage explants caused exaggerated mechanical strain (29) and depletion in bovine cartilage explants resulted in increased chondrocyte apoptosis following mechanical loading and movement (28). Genetic deficiency of Prg4, which increases cartilage surface friction and causes wear (30), also leads to a progressive loss of chondrocytes (31).

Protection from cartilage damage following DMM surgery has been reported in genetically engineered mice that could not express enzymes such as ADAMTS4, ADAMTS5 or MMP13 in cartilage $(17,18)$. These findings imply that before DMM induces cell death, it induces chondrocytes to produce factors that facilitate cartilage destruction. We directly tested this hypothesis by comparing the effect of DMM surgery in mice with and without (i.e., DTA ablated) living articular surface chondrocytes (Figure 5). Mice whose chondrocytes had been DTA ablated before DMM surgery had significantly less cartilage damage, as assessed by Osteoarthritis Research Society International (OARSI) scoring (19), than control mice. These data indicate that dead chondrocytes do less harm after DMM surgery.

We are not the first to apply confocal imaging to articular cartilage $(10,32-36)$. We believe that the present study is novel in its demonstration of the utility of confocal imaging for quantifying several useful measures of cartilage structure/organization, including cartilage volume, chondrocyte number, cells/volume near the surface, cell division frequency, and nearest neighbor distance. Although there are differences in the coefficients of variation (CVs) for these measurements between articular cartilage surfaces (e.g., femoral head and humeral head), most CVs were still sufficiently small ( $<10 \%$ ) (Supplemental Table 2$)$ so that statistically significant differences could be observed with small sample sizes (Supplemental Table 3). For example, confocal microscopy provided $90 \%$ power to detect a $30 \%$ difference in superficial chondrocyte density using 4 specimens per group for the femoral head, humeral head, and lateral femoral condyle and 6 specimens per group for the medial femoral condyle (Supplemental Table 3). At present, the confocal imaging method is limited to ex vivo analyses of cartilage structure, a limitation shared with other highresolution imaging techniques such as $\mu \mathrm{CT}$ and $\mu \mathrm{MRI}(37,38)$.

Compared with other methods, imaging articular cartilage using confocal microscopy is fast. Specimen processing requires little hands-on time and can be completed within 72 hours; this contrasts with standard histologic methods that require decalcification of mineralized tissue, formalin fixation, paraffin embedding, sectioning, and staining before microscopic imaging can be performed. Specimens prepared for confocal imaging can be stored for at least 3 weeks without any significant changes in measurements. Data acquisition was also quick. Obtaining $2-\mu \mathrm{m}$ interval images 
spanning a volume of $760 \mu \mathrm{m} \times 760 \mu \mathrm{m} \times 50 \mu \mathrm{m}$, for 4 separate fluorophores (DAPI, Alexa Fluor 647, dTomato, and eGFP) required approximately 30 minutes per specimen. The time to obtain micron level resolution using confocal microscopy was shorter than in similar micron-scale imaging with $\mu \mathrm{CT}$ or $\mu \mathrm{MRI}$, each of which requires several hours per specimen $(37,39-41)$. The applications described here used confocal microscopy to measure cell number, cell density, and cell division. Other fluorophores could be used to measure other aspects of cartilage structure and chondrocyte identity, such as extracellular matrix volume and protein expression.

In sum, employing confocal microscopy, standard histology, genetically engineered mice, and DMM surgery, we show that chondrocyte death is not a proximate cause of cartilage damage when the death has left the cartilage surface intact. We further show that, while DMM surgery causes chondrocyte death, it is the dysfunction of chondrocytes prior to their demise that enhances cartilage damage in the DMM model. Thus, posttraumatic osteoarthritis, as occurs following injury (42), is the likely result of initial cartilage damage that predisposes chondrocytes to adopt a catabolic phenotype. Therapies that prevent this chondrocytemediated cartilage catabolism after injury should reduce the burden of degenerative joint disease.

\section{Methods}

Experimental animals and procedures. Wild-type C57BL/6J mice, $R 26^{\text {DTA }}$ mice with a C57BL/6J background, $R 26^{m T m G}$ mice with a 129X1/SvJ background, and Prg $4^{\text {GFPCreERt2 }}$ mice were obtained from the Jackson Laboratory (stock numbers 000664, 009669, 007576, and 022757, respectively).

Cre-recombinase activity in postnatal mice with the $\operatorname{Prg} 4^{\text {CreERt } 2}$ allele was induced by administering $100 \mathrm{mg} / \mathrm{kg}$ tamoxifen diluted in corn oil (both from Sigma-Aldrich) for 10 consecutive days by i.p. injection; i.p. injections of corn oil alone served as a negative control. At least 5 animals, including both sexes per genotype, treatment, and age group were studied.

To label dividing cells, EdU (Invitrogen) was given for 10 consecutive days to 40 -day-old mice $(50 \mathrm{mg} / \mathrm{kg} / \mathrm{dose}$ via i.p.) or for 30 consecutive days to 2 -month-old mice $(0.3 \mathrm{mg} / \mathrm{ml}$ in drinking water).

DMM was performed as previously described $(22,26)$. Briefly, 9to 11-week-old mice were anesthetized and their right knees were prepared for aseptic surgery. The joint capsule immediately medial to the patellar tendon was opened. The intercondylar region was exposed to visualize the meniscotibial ligament. This ligament was cut, after which the joint capsule and skin were closed. Sham-surgery animals had the joint capsule opened without cutting the meniscotibial ligament. The knees from the animals were studied at 4 and 12 weeks after surgery. DTA ablation, prior to performing DMM or sham surgery, was accomplished by administering tamoxifen for 10 consecutive days at from 21 to 30 days of age and for another 5 consecutive days, ending 1 week before surgery was performed.

Sample preparation. Mice were euthanized by $\mathrm{CO}_{2}$ inhalation. Specimens consisting of whole humeri and femora were removed, cleaned of muscle and ligament, placed in approximately $1 \mathrm{ml}$ of PBS containing $4 \%$ paraformaldehyde (PFA) (Affymetrix), and stored at $4^{\circ} \mathrm{C}$.

Specimens consisting of knee joints with intact synovial capsules were fixed in $4 \%$ PFA for more than 72 hours, decalcified for 14 days in $14 \%$ EDTA ( $\mathrm{pH} 7.5)$, and embedded in paraffin.
DAPI and EdU staining. Cell nuclei in the fixed humeri and femora were stained by adding DAPI dye $(10 \mu \mathrm{g} / \mathrm{ml})$ to the $4 \%$ PFA solution for more than 16 hours. Unincorporated dye was removed by washing specimens twice for 15 minutes in $4 \%$ PFA.

For animals that had been given EdU in vivo to label dividing cells, the EdU-containing cells in the 4\% PFA-fixed humeri and femora were detected using the Alexa Fluor 647 Click-iT EdU Imaging Kit (Invitrogen). The 4\% PFA fixed small intestine specimens were used as positive controls. The fixed tissues were subjected to two 15-minute washes with 3\% BSA (Sigma-Aldrich) in PBS, permeabilized by being placed in 2\% Triton X-100 (Sigma-Aldrich) in PBS for 2 hours at room temperature, and washed again with two 15 -minute washes in $3 \%$ BSA in PBS. Click-iT reaction cocktail was prepared according to the manufacturer's instructions and applied to the tissues for 24 hours at $4^{\circ} \mathrm{C}$. Specimens were given two 30 -minute washes in 3\% BSA in PBS, returned to $4 \%$ PFA solution, and then stained with DAPI dye.

Confocal laser scanning. Each individual specimen was placed on a glass-bottom plate and immersed in PBS in the orientation indicated (Supplemental Figure 1A). The cartilage surface in contact with the glass was imaged at 1- or 2- $\mu \mathrm{m}$ intervals to a depth of $50 \mu \mathrm{m}$ using an inverted Zeiss LSM780 confocal laser-scanning microscopy system (Carl Zeiss) (Supplemental Figure 1B). The objective used was Zeiss $\times 20$ Plan Apochromat NA 0.8 WD 550 mm dry. The confocal imaging was controlled through the software ZEN 2009 from Zeiss. The frame size of the image was $760 \mu \mathrm{m} \times 760 \mu \mathrm{m}$. A Z-stack $50-\mu \mathrm{m}$ thick for femoral heads and femoral condyles or $60-\mu \mathrm{m}$ thick for humeral heads ( 1 or $2 \mu \mathrm{m} /$ slice) was projected from the surface with a speed of 6. Samples were labeled with up to 4 fluorophores including DAPI, eGFP, dTomato, and Alexa Fluor 647. Excitation wavelengths used were $358 \mathrm{~nm}$ for DAPI, $488 \mathrm{~nm}$ for eGFP, $561 \mathrm{~nm}$ for dTomato, and $647 \mathrm{~nm}$ for Alexa Fluor 647. Emission wavelengths used were 385 to $500 \mathrm{~nm}$ for DAPI, 500 to $555 \mathrm{~nm}$ for eGFP, 585 to $650 \mathrm{~nm}$ for dTomato, and 650 to $750 \mathrm{~nm}$ for Alexa Fluor 647. Gains were 700 for DAPI, 800 for eGFP, 750 for dTomato, and 800 for Alexa Fluor 647.

$3 D$ reconstruction of the articular cartilage and quantitative analysis. The imaging software IMARIS was used to perform 3D image reconstruction and calculate chondrocyte number and density (43). The spots function within IMARIS was used to obtain the center of mass in each cell nucleus and count the number of nuclei with a specified radius (Figure 1A). The radii of the DAPI- and EdU-labeled cell nuclei were set at $5 \mu \mathrm{m}$, and the radii of membrane-bound eGFP and dTomato fluorescence-labeled cells were set at $10 \mu \mathrm{m}$. Cell nuclei were counted in a specified region within the image (Figure 1, A, C, E, and F). The surface function was used to compute the volume of cartilage surface (Figure 1, B and D). See Supplemental Methods for detailed information.

DAPI and EdU staining of chondrocyte nuclei were also analyzed for chondrocyte arrangement and clustering. Through IMARIS, the centroid of each chondrocyte nucleus was plotted on an arbitrary $3 \mathrm{D}$ coordinate field. These coordinates were then imported into MATLAB (MathWorks). A custom MATLAB script was developed to determine the nearest neighbor distance for each cell nucleus.

Histologic analysis. The 4\% PFA-fixed, 14\% EDTA-decalcified, and paraffin-embedded intact knee joints were sectioned in the sagittal plane and stained with H\&E or Safranin O and Fast Green (Thermo Scientific). Sections from equivalent regions of the joint were obtained in order to compare findings between animals. OARSI scoring was performed as previously described (19) on coronal sections of paired 
femoral condyles and tibial plateaus. Six independent observers, who were blind to whether the joint had had DTA ablation or DDM surgery, scored the sections. Medial and lateral femoral condyle and medial and lateral tibial plateau scores were summed for each animal, and the averages of the 6 independent observers' summed scores were used in the statistical analyses.

Statistics. Bland-Altman paired analyses with $95 \%$ CIs were performed to compare confocal microscopy measurements obtained for each mouse's left and right femoral head, humeral head, lateral condyle, and medial condyle in order to assess agreement and correlation. A 1-sample $t$ test was used to supplement the analysis on agreement using the difference in the measurements between the left and right sides. Repeated measures ANOVA using generalized estimating equations (GEE) followed by a 1-sided $t$ test was used to assess the impact of time in $4 \%$ PFA on each of the 4 primary measures for femoral head, humeral head, lateral femoral condyle, and medial femoral condyle. $\mathrm{CV}$ was determined to assess differences between variability in relation to the calculated mean for each scan or time point. Sample size estimates were calculated based on an independent Student's $t$ test with equal sample sizes for each percentage change, and $t$ tests were used to assess statistical significance for confocal microscopy measurements between DTA-ablated and control joints. Comparison of chondrocyte nearest neighbor distances in control and DTA-ablated mice was performed using Student's $t$ test, and variability of histogram shape was assessed according to Levene's test. The nonparametric Wilcoxon rank-sum test was used to compare OARSI scores between mice that did or did not experience DTA ablation and DMM surgery.
Two-tailed $P$ values of less than 0.05 were considered significant. Two articular cartilage surfaces were damaged when they were being dissected from mice and were not included in the confocal microscopy and histologic assessments.

Study approval. The Institutional Animal Care and Use Committees at the Boston Children's Hospital and the Harvard School of Dental Medicine approved these studies.

\section{Author contributions}

MZ, GDJ, and MLW conceived the research. MZ, SBM, and YH performed confocal imaging and analysis, EdU incorporation, and DTA-induction experiments. MZ, LX, and YL performed DMM experiments. AMH and DZ performed statistical analyses.

\section{Acknowledgments}

The authors thank Nandan Nerurkar, Hunter Elliott, Evelyn Fly$\mathrm{nn}$, Jim Koepfler, and Yajun Cui and Jakob Sieker for help with confocal microscopy, the Imaris software package, histology, specimen photography, and OARSI scoring, respectively. The authors also thank the anonymous peer reviewers of this manuscript for their constructive comments. This work was supported by NIH grant AR050180 (to G.D. Jay and M.L. Warman) and the Howard Hughes Medical Institute.

Address correspondence to: Matthew L. Warman, 320 Longwood Avenue, Enders 250, Boston, Massachusetts 02115, USA. Phone: 617.919.2371; E-mail: Matthew.Warman@childrens.harvard.edu.
1. Pearle AD, Warren RF, Rodeo SA. Basic science of articular cartilage and osteoarthritis. Clin Sports Med. 2005;24(1):1-12.

2. Buckwalter JA, Mankin HJ, Grodzinsky AJ. Articular cartilage and osteoarthritis. Instr Course Lect. 2005;54:465-480.

3. Johnson VL, Hunter DJ. The epidemiology of osteoarthritis. Best Pract Res Clin Rheumatol. 2014;28(1):5-15.

4. Neogi T, Zhang Y. Epidemiology of osteoarthritis. Rheum Dis Clin North Am. 2013;39(1):1-19.

5. Roos EM. Joint injury causes knee osteoarthritis in young adults. Curr Opin Rheumatol. 2005;17(2):195-200.

6. Scott DL, Wolfe F, Huizinga TW. Rheumatoid arthritis. Lancet. 2010;376(9746):1094-1108.

7. Weinstein AM, et al. Estimating the burden of total knee replacement in the United States. J Bone Joint Surg Am. 2013;95(5):385-392.

8. Grogan SP, D'Lima DD. Joint aging and chondrocyte cell death. Int JClin Rheumtol. 2010;5(2):199-214.

9. Martin JA, Buckwalter JA. Aging, articular cartilage chondrocyte senescence and osteoarthritis. Biogerontology. 2002;3(5):257-264.

10. Cao Y, et al. Proinflammatory cytokines stimulate mitochondrial superoxide flashes in articular chondrocytes in vitro and in situ. PLoS One. 2013;8(6):e66444.

11. Loeser RF. Aging and osteoarthritis: the role of chondrocyte senescence and aging changes in the cartilage matrix. Osteoarthr Cartil. 2009;17(8):971-979.

12. Colwell CW Jr, et al. In vivo changes after mechanical injury. Clin Orthop Relat Res. 2001;(391 suppl):S116-S123.

13. Duda GN, Eilers M, Loh L, Hoffman JE, Kääb M, Schaser K. Chondrocyte death precedes structural damage in blunt impact trauma. Clin Orthop Relat Res. 2001;(393):302-309.

14. Seol D, et al. Chondrogenic progenitor cells respond to cartilage injury. Arthritis Rheum. 2012;64(11):3626-3637.

15. Poulet B, et al. Time-series transcriptional profiling yields new perspectives on susceptibility to murine osteoarthritis. Arthritis Rheum. 2012;64(10):3256-3266.

16. Burleigh A, et al. Joint immobilization prevents murine osteoarthritis and reveals the highly mechanosensitive nature of protease expression in vivo. Arthritis Rheum. 2012;64(7):2278-2288.

17. Little $\mathrm{CB}$, et al. Matrix metalloproteinase 13-deficient mice are resistant to osteoarthritic cartilage erosion but not chondrocyte hypertrophy or osteophyte development. Arthritis Rheum. 2009;60(12):3723-3733.

18. Majumdar MK, et al. Double-knockout of ADAMTS- 4 and ADAMTS- 5 in mice results in physiologically normal animals and prevents the progression of osteoarthritis. Arthritis Rheum. 2007;56(11):3670-3674.

19. Glasson SS, Chambers MG, Van Den Berg WB, Little CB. The OARSI histopathology initiative recommendations for histological assessments of osteoarthritis in the mouse. Osteoarthr Cartil. 2010;18(suppl 3):S17-S23.

20. Kozhemyakina E, et al. Identification of a prg4-expressing articular cartilage progenitor cell population in mice. Arthritis Rheumatol. 2015;67(5):1261-1273.

21. Voehringer D, Liang HE, Locksley RM. Homeostasis and effector function of lymphopenia-induced "memory-like" $\mathrm{T}$ cells in constitutively T cell-depleted mice. JImmunol. 2008;180(7):4742-4753.

22. Glasson SS, Blanchet TJ, Morris EA. The surgical destabilization of the medial meniscus (DMM) model of osteoarthritis in the 129/SvEv mouse. Osteoarthr Cartil. 2007;15(9):1061-1069.

23. Henry SP, Liang S, Akdemir KC, de Crombrugghe B. The postnatal role of Sox 9 in cartilage. J Bone Miner Res. 2012;27(12):2511-2525.

24. Loty B, Courpied JP, Tomeno B, Kerboull M, Postel M, Forest M. [Massive bone allografts sterilized by irradiation: evaluation following 5 years of use]. Acta Orthop Belg. 1991;57(suppl 2):35-43.

25. Parsch D, Brümmendorf TH, Richter W, Fellenberg J. Replicative aging of human articular chondrocytes during ex vivo expansion. Arthritis Rheum. 2002;46(11):2911-2916.

26. Xu L, et al. Attenuation of osteoarthritis progression by reduction of discoidin domain receptor 2 in mice. Arthritis Rheum. 2010;62(9):2736-2744.

27. Ryu JH, Shin Y, Huh YH, Yang S, Chun CH, Chun JS. Hypoxia-inducible factor- $2 \alpha$ regulates Fasmediated chondrocyte apoptosis during osteoarthritic cartilage destruction. Cell Death Differ. 2012;19(3):440-450.

28. Waller KA, Zhang LX, Elsaid KA, Fleming BC, Warman ML, Jay GD. Role of lubricin and boundary lubrication in the prevention of chondrocyte apoptosis. Proc Natl Acad Sci US A. 
2013;110(15):5852-5857.

29. Wong BL, Bae WC, Chun J, Gratz KR, Lotz M, Sah RL. Biomechanics of cartilage articulation: effects of lubrication and degeneration on shear deformation. Arthritis Rheum. 2008;58(7):2065-2074.

30. Jay GD, et al. Association between friction and wear in diarthrodial joints lacking lubricin. Arthritis Rheum. 2007;56(11):3662-3669.

31. Karamchedu NP, Tofte JN, Waller KA, Zhang LX, Patel TK, Jay GD. Superficial zone cellularity is deficient in mice lacking lubricin: a stereoscopic analysis. Arthritis Res Ther. 2015;18(1):64.

32. Verschure PJ, Van Marle J, Van Noorden CJ, Van den Berg WB. The contribution of quantitative confocal laser scanning microscopy in cartilage research: chondrocyte insulin-like growth factor-1 receptors in health and pathology. Microsc Res Tech. 1997;37(4):285-298.

33. Zelenski NA, et al. Type VI collagen regulates pericellular matrix properties, chondrocyte swelling, and mechanotransduction in mouse articular cartilage. Arthritis Rheumatol.
2015;67(5):1286-1294.

34. Jablonski CL, Ferguson S, Pozzi A, Clark AL. Integrin $\alpha 1 \beta 1$ participates in chondrocyte transduction of osmotic stress. Biochem Biophys Res Commun. 2014;445(1):184-190.

35. Stok KS, Müller R. Morphometric characterization of murine articular cartilage - novel application of confocal laser scanning microscopy. Microsc Res Tech. 2009;72(9):650-658.

36. Wong M, Wuethrich P, Eggli P, Hunziker E. Zonespecific cell biosynthetic activity in mature bovine articular cartilage: a new method using confocal microscopic stereology and quantitative autoradiography. JOrthop Res. 1996;14(3):424-432.

37. Ruan MZ, Dawson B, Jiang MM, Gannon F, Heggeness M, Lee BH. Quantitative imaging of murine osteoarthritic cartilage by phase-contrast micro-computed tomography. Arthritis Rheum. 2013;65(2):388-396.

38. Tremoleda JL, Khalil M, Gompels LL, Wylezinska-Arridge M, Vincent T, Gsell W. Imaging technologies for preclinical models of bone and joint disorders. EJNMMI Res. 2011;1(1):11.

39. Shapiro EM, Skrtic S, Sharer K, Hill JM, Dunbar CE, Koretsky AP. MRI detection of single particles for cellular imaging. Proc Natl Acad Sci US A. 2004;101(30):10901-10906.

40. Ullmann JF, Cowin G, Kurniawan ND, Collin SP. Magnetic resonance histology of the adult zebrafish brain: optimization of fixation and gadolinium contrast enhancement. NMR Biomed. 2010;23(4):341-346.

41. Xie L, Lin AS, Levenston ME, Guldberg RE. Quantitative assessment of articular cartilage morphology via EPIC-microCT. Osteoarthr Cartil. 2009;17(3):313-320.

42. Anderson DD, et al. Post-traumatic osteoarthritis: improved understanding and opportunities for early intervention. J Orthop Res. 2011;29(6):802-809.

43. Combes AN, Short KM, Lefevre J, Hamilton NA, Little MH, Smyth IM. An integrated pipeline for the multidimensional analysis of branching morphogenesis. Nat Protoc. 2014;9(12):2859-2879. 